\title{
An experimental and theoretical examination of the effect of sulfur on the pyrolytically grown carbon nanotubes from sucrose-based solid state precursors
}

\author{
Gokce Kucukayan ${ }^{a}$, Rasim Ovali ${ }^{b}$, Serim Ilday ${ }^{a}$, Beril Baykal ${ }^{c}$, Hilmi Yurdakul ${ }^{d}$, \\ Servet Turan ${ }^{d}$, Oguz Gulseren ${ }^{b}$, Erman Bengu ${ }^{c, *}$ \\ a Bilkent University, Institute of Engineering and Science, Material Science and Nanotechnology Graduate Program, Bilkent, \\ 06800 Ankara, Turkey \\ b Bilkent University, Department of Physics, Bilkent, 06800 Ankara, Turkey \\ c Bilkent University, Department of Chemistry, Bilkent, 06800 Ankara, Turkey \\ d Anadolu University, Department of Materials Science and Engineering, Eskisehir, Turkey
}

\section{A R T I C L E I N F O}

Article history:

Received 23 June 2010

Accepted 24 September 2010

Available online 1 October 2010

\begin{abstract}
A B S T R A C T
Multi-walled carbon nanotubes (MWCNTs) were synthesized through pyrolysis of the sulfuric acid-carbonized byproduct of sucrose. While the presence of sulfur in the reaction media has a key role in the formation and population density of MWCNTs, we have not observed the formation of Y-junctions or encountered other novel carbon nanotube formations. Results indicate the presence of sulfur in catalyst particles trapped inside nanotubes, but failed to find sulfur in the side-walls of the CNTs. In order to verify and explain these findings, we analyzed the behavior of sulfur and its possible effects on the side-wall structure of CNTs by using density functional theory-based calculations on various atomic models depicting sulfur inclusion in the side-walls. The results of the computational study were in line with the experimental results and also provided a new perspective by suggesting that the defects such as pentagons may act as nucleation sites for the Y-branches. The results indicated that sulfur prefers to adsorb on these defective regions, but it is not responsible for the formation of these structures or defects.
\end{abstract}

(c) 2010 Elsevier Ltd. All rights reserved.

\section{Introduction}

The last two decades witnessed great advances in the synthesis and functionalization of carbon nanotubes (CNTs), however fabrication of two- (2D) or three-dimensional (3D) networks, and/or lattices of these still remain as a major challenge. A robust network requires strong bonding between the building blocks. For a 3D network of CNTs, this can be achieved by "welding" of CNTs to each other through covalent bonding [1]. The junctions formed by welding provoke further interest, especially the three terminal "Y" junctions of CNTs.
It is proposed that these could be potentially used in the fabrication of electronic devices [2-5]. Analysis of the junction geometry at an atomic scale indicates the requirement for the use of non-hexagonal carbon rings [1]. Similarly, nonhexagonal boron nitride (BN) rings were also used for the modeling of bridging between BN nanotubes and fullerenes for the assembly of experimentally observed complex BN nanostructures by Bengu and Marks [6]. With regards to the formation of "Y" junctions, several studies argued a key catalytic role for sulfur during synthesis; however no supporting data was presented [7-11]. Only recently, presence of sulfur

* Corresponding author: Fax: +90 3122664068.

E-mail address: bengu@fen.bilkent.edu.tr (E. Bengu).

0008-6223/\$ - see front matter (c) 2010 Elsevier Ltd. All rights reserved. doi:10.1016/j.carbon.2010.09.050 
was claimed through evidence from analytical electron microscopy in the vicinity of CNT branches [12]. In the same study, an atomic model explaining the required curvature for branching mechanism was also proposed. Ab-initio density functional theory (DFT) calculations based on this model with sulfur inclusions were then presented as supporting evidence for the potential role of sulfur in the formation of Yjunctions.

In this report, we present the results of our work on the pyrolysis of carbonized sucrose residue in sulfur rich environment for the fabrication of CNTs. Sucrose is a carbon-rich compound which can be easily carbonized in the presence of dehydrating acids (carbonization) [13], however sucrose is considered to be a non-graphitizing carbon [14]. There have been reports of fullerene-like structures [15] and multi walled carbon nanotube (MWCNT) formation [16] through annealing of charred sucrose with boron powder in an inert atmosphere in excess of $2200^{\circ} \mathrm{C}$. There are other solid state precursors used for synthesis of CNTs such as camphor and various other metallocenes [17]. While sucrose impregnated mesoporous silicates have been also used to grow MWCNTs [18], to best of our knowledge there is no other work in the literature systematically investigating the effect of sulfur on CNT morphology through a solid-state synthesis route. Finally, we present a theoretical understanding of Y-branch formation and define conditions favoring incorporation of sulfur to CNT structure using first principles density functional calculations.

\section{Experimental}

\subsection{Synthesis of CNTs through pyrolysis}

All reagents and solvents were purchased from SigmaAldrich and used without further purification. In the first step of CNT preparation, $5 \mathrm{~g}$ of sucrose and $25 \mathrm{mg}$ of catalyst were mixed and ground to a fine powder using an alumina mortar and pestle. As for catalyst, we have used several different mixtures; 1:1 mixture of powdered $\mathrm{Fe}(\geqslant 99.9 \%)$ and $\mathrm{Ni}$ ( $\geqslant 99.9 \%$ ) with average particle size $<1 \mu \mathrm{m}$, iron (II) sulfate $\left(\mathrm{FeSO}_{4} \cdot 7 \mathrm{H}_{2} \mathrm{O}, \geqslant 99.0 \%\right)$ or iron sulfide ( $\left.\mathrm{FeS}, \geqslant 99.9 \%\right)$. Then, $5 \mathrm{ml}$ solution of varying amounts of sulfuric acid $\left(\mathrm{H}_{2} \mathrm{SO}_{4}\right.$, $\geqslant 99.999 \%)$ and phosphoric acid $\left(\mathrm{H}_{3} \mathrm{PO}_{4}, 85 \%\right.$ in $\left.\mathrm{H}_{2} \mathrm{O}\right)$ were added to this mixture. Hence, as these acids are strong dehydrating agents, the reaction between the acid solution and sucrose-catalyst mixture resulted in the carbonization of the sucrose component. In the final step, the carbonized aggregate/residue from acid dehydration was pyrolyzed in an atmosphere controlled furnace at $1330^{\circ} \mathrm{C}$ for $4 \mathrm{~h}$ under flowing Ar atmosphere (99.999\%). Purified MWCNTs for Raman spectroscopy were prepared by heating the product of pyrolysis in a tube furnace for $6 \mathrm{~h}$ at $600{ }^{\circ} \mathrm{C}$ in air. A summary of the experiments performed in this study is provided in Table 1.

\subsection{Characterization}

Scanning electron microscopy (SEM) imaging of the CNTs in the pyrolysis residue was performed on a Carl-Zeiss EVO 40 $\left(\mathrm{LaB}_{6}\right.$ filament). For the calculation of areal density and aspect ratio for MWCNTs, we have analyzed as much as 20 randomly acquired SEM images per run. Transmission electron microscopy (TEM) and high angle annular dark field imaging (HAADF) of the CNTs were done using a JEOL (JEM-2100 F) microscope operating at $200 \mathrm{kV}$. Chemical analysis of the same CNT samples were performed inside the TEM through energy dispersive spectroscopy (EDS, JEOL JED-2300T) and electron energy loss spectroscopy (EELS, GATAN Tridiem). The Raman spectra from the post-pyrolysis residue were recorded with a Horiva (Jobin-Yvon MicroRaman) spectrometer. The source of radiation was a laser operating at a wavelength of $632.8 \mathrm{~nm}$ and a power of $25 \mathrm{~mW}$.

\subsection{Density functional calculations}

In order to understand the effect of sulfur on CNT morphology, we have performed the first principles plane-wave calculations [19] within DFT $[20,21]$ by the projector-augmented-wave (PAW) potentials [22,23] using Vienna ab-initio simulation package (VASP) program [24-26]. The exchange-correlation potential was expressed in terms of the generalized gradient approximation (GGA) (Perdew-Wang 91 type) [27]. A planewave cutoff energy of $500 \mathrm{eV}$ was used in all calculations. A large graphene supercell with $10 \AA$ of vacuum is introduced to minimize the ion-ion interaction in the non-periodic directions. Therefore, only the $\Gamma$ point is used as k-point sampling in the Brillouin zone [28]. The partial occupancy around the Fermi level is treated by Gaussian smearing with a smearing parameter of $0.08 \mathrm{eV}$. For all calculations energy was converged to within $10^{-4} \mathrm{eV}$ accuracy. In all calculations, edges of finite sized structures are saturated by hydrogen atoms and then all of the atoms are relaxed to their minimum energy configurations by using conjugate gradient method where total energy and atomic forces are minimized. Maximum force magnitude remained on each atom is set at most to $0.06 \mathrm{eV} / \AA$.

\section{Results and discussion}

The final product of the pyrolysis step was found to be composed of an amorphous carbon matrix with randomly scattered MWCNTs. SEM micrograph, in Fig. 1a, shows a typical post-pyrolysis residue from $\mathrm{H}_{2} \mathrm{SO}_{4}$ dehydrated sucrosecatalyst mixture; a dense bundle made out of MWCNTs of various sizes. The inset shows multiple catalyst particles trapped inside one of the MWCNTs. We believe that this is because during the pyrolysis process, temperature of the furnace $\left(1330^{\circ} \mathrm{C}\right)$ was kept well above the Fe-S eutectic temperature

\section{Table 1 - Complete list of catalyst powder and dehydrating} acid used during the synthesis experiments.

\begin{tabular}{|c|c|c|c|}
\hline \multirow[t]{2}{*}{ Catalyst type } & \multicolumn{3}{|c|}{ Dehydration acid type } \\
\hline & $\mathrm{H}_{2} \mathrm{SO}_{4}$ & $\mathrm{H}_{3} \mathrm{PO}_{4}$ & $\begin{array}{c}\text { No dehydration } \\
\text { acid }\end{array}$ \\
\hline $\mathrm{Fe} / \mathrm{Ni}$ & Yes $^{\mathrm{a}}$ & $\mathrm{No}^{\mathrm{b}}$ & No \\
\hline $\mathrm{FeSO}_{4} \cdot 7 \mathrm{H}_{2} \mathrm{O}$ & Yes & Yes & No \\
\hline $\mathrm{FeS}$ & Yes & Yes & No \\
\hline
\end{tabular}



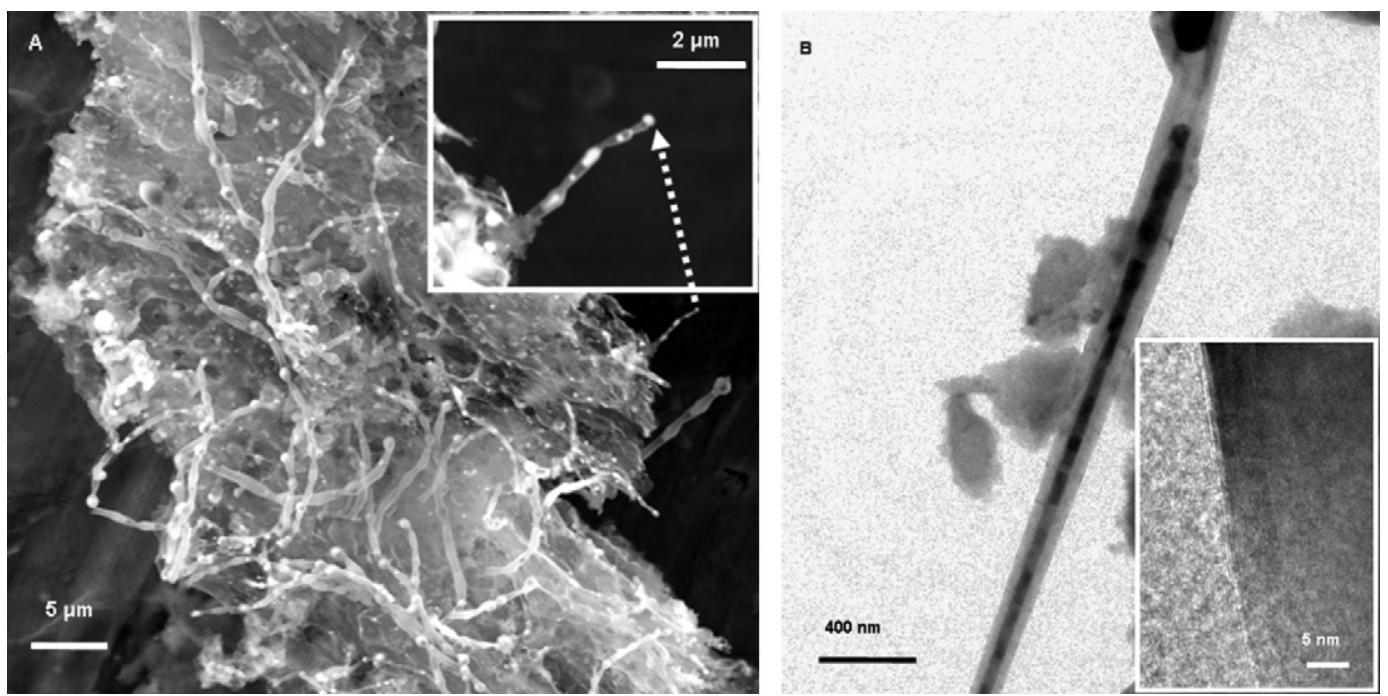

Fig. 1 - (a) SEM image of sucrose based final product displays randomly distributed MWCNTs together with an amorphous carbon matrix. The inset reveals multiple catalyst particles trapped in the hollow core of the MWCNT. (b) Bright field S/TEM image of MWCNT provides further detail on the morphology and catalyst particle. The inset shows the graphitic side walls of MWCNT.

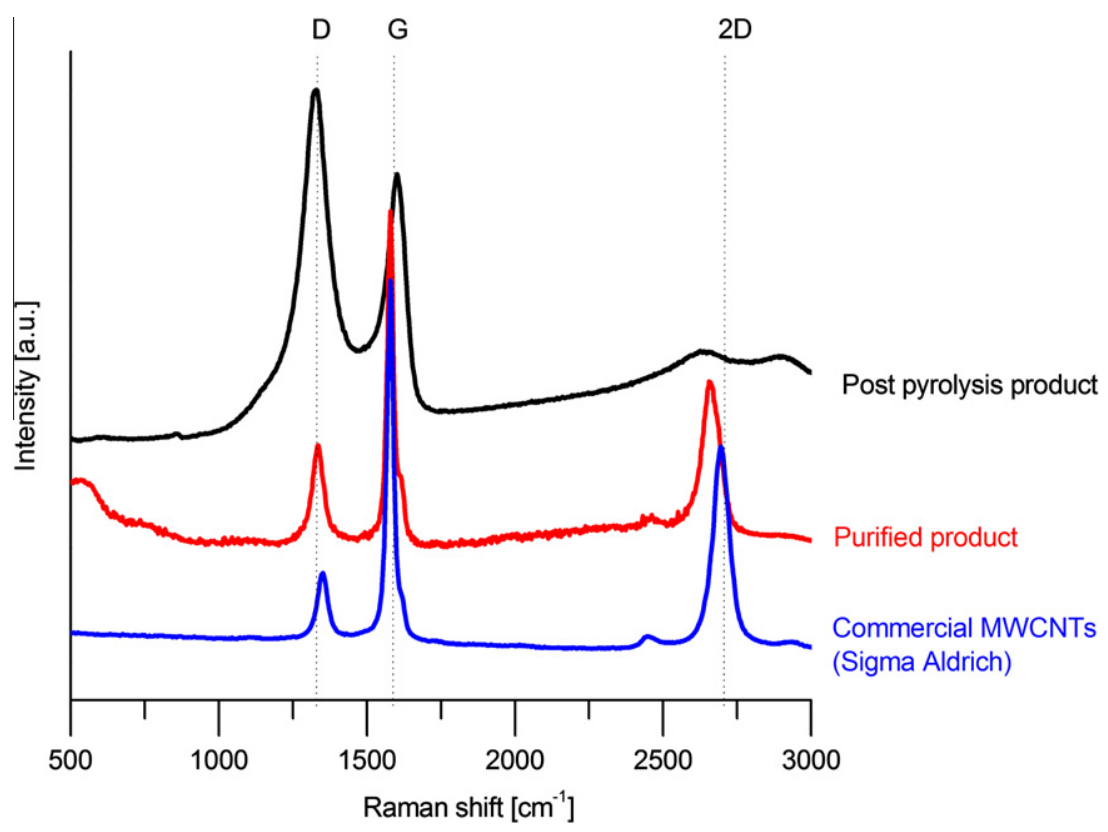

Fig. 2 - Comparison of the Raman spectra from as-synthesized, purified and commercial MWCNTs.

$\left(988^{\circ} \mathrm{C}\right)$ and hence, the catalyst particles were at a molten state and had high capillary mobility. As a result, MWCNTs with completely metal filled cores were also observed; in Fig. $1 \mathrm{~b}$ bright field S/TEM image of one is given, the inset shows the well-formed side-wall structure of a MWCNT synthesized in this study. Furthermore, Fig. 2 shows the Raman spectrum from synthesized MWCNTs in this study after purification versus commercially available material. D (disorder mode) and G (tangential stretch mode) peak shapes and positions in the spectrum from the purified product match to those from commercially available material which confirms presence of MWCNTs.
In our experiments, we have observed MWCNTs only in samples prepared with either sulfur containing additives or those prepared with $\mathrm{H}_{2} \mathrm{SO}_{4}$ containing acid dehydration. We have not observed presence of CNTs inside the post-pyrolysis products of pure $\mathrm{H}_{3} \mathrm{PO}_{4}$ dehydrated sucrose-catalyst mixtures. We further scrutinized this observation by replacing $\mathrm{Fe}$ and $\mathrm{Ni}$ catalyst mixture first with $\mathrm{FeSO}_{4} \cdot 7 \mathrm{H}_{2} \mathrm{O}$ and then with FeS. As shown in Fig. 3a and b, usage of both $\mathrm{FeSO}_{4} \cdot 7 \mathrm{H}_{2} \mathrm{O}$ and $\mathrm{FeS}$ as replacement catalysts while $\mathrm{H}_{3} \mathrm{PO}_{4}$ was used for carbonization, resulted in the formation of CNTs. These findings indicated that sulfur has a critical role in the growth of CNTs synthesized by this technique. Moreover, we have not 

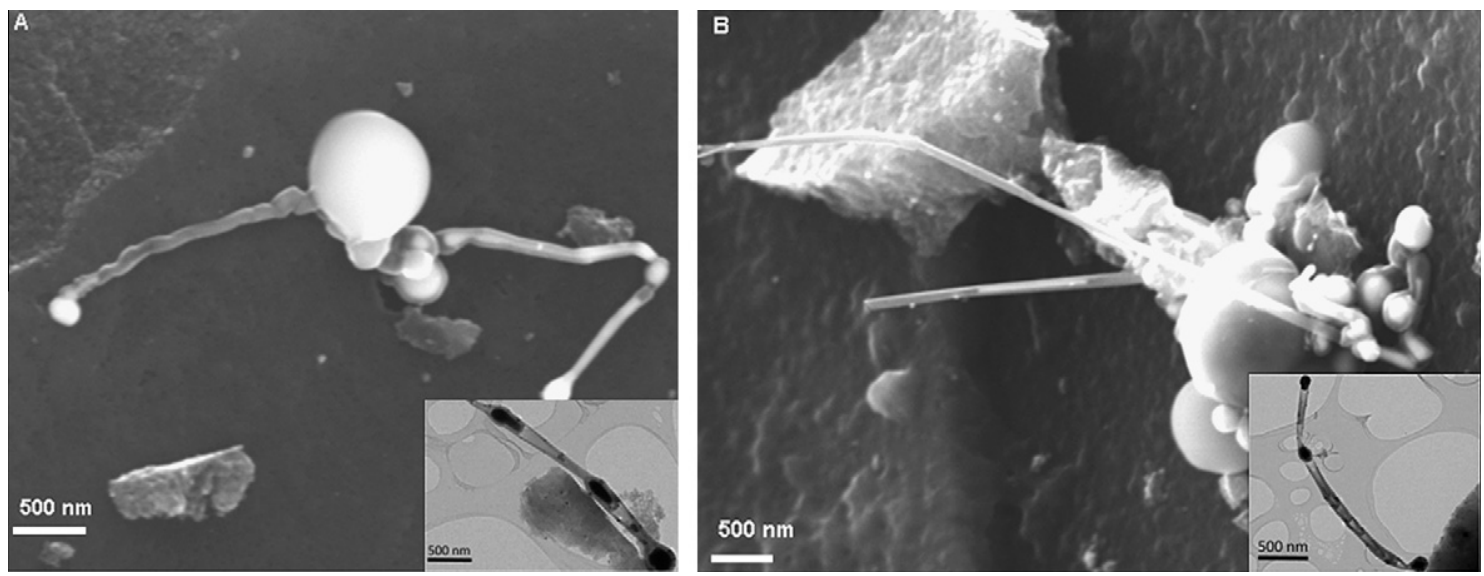

Fig. 3 - SEM images of MWCNTs synthesized by the pyrolysis of (a) $\mathrm{H}_{3} \mathrm{PO}_{4}$ carbonized sucrose-FeSO $\mathrm{O}_{4} \cdot 7 \mathrm{H}_{2} \mathrm{O}$ mixture and (b) $\mathrm{H}_{3} \mathrm{PO}_{4}$ carbonized sucrose-FeS mixture. Insets show TEM images from respective samples.

observed presence of CNTs in samples pyrolyzed at a temperature below $1200{ }^{\circ} \mathrm{C}$ at all regardless of acid used for carbonization and catalyst. The effect of sulfur on the synthesis of various carbons has been known for decades, and sulfur has long been viewed as a graphitization agent [30]. Kim et al. [31] reported a significant increase in the density of filamentous carbon formed by the addition of $\mathrm{H}_{2} \mathrm{~S}$ in the reaction system up to $500 \mathrm{ppm}$. Furthermore, Tibbetts et al. [32] reported considerable improvement in the catalytic activity of Fe for the production of carbon fibers.

To explore the effect of sulfur on MWCNT synthesis in the pyrolysis route we have detailed above, we first compared the Raman spectra from $\mathrm{H}_{2} \mathrm{SO}_{4}$ and $\mathrm{H}_{3} \mathrm{PO}_{4}$ carbonized aggregates, as shown in Fig. 4. The similarity between the Raman spectra from the two dehydrated mixtures taken before pyrolysis suggested that the post carbonization aggregates were analogous to each other. Relative intensity ratios and positions of $\mathrm{D}$ and $\mathrm{G}$ bands are listed the inset which clearly implies small inplane correlation lengths for the graphitic networks in both carbonized aggregates [29]. In short, regardless of whether $\mathrm{H}_{3} \mathrm{PO}_{4}$ or $\mathrm{H}_{2} \mathrm{SO}_{4}$ was used during the dehydration, carbonization step converted sucrose-catalyst mixture into a disordered carbonaceous aggregate. Hence, in order to further explore and provide a satisfactory explanation, we studied the effect of sulfur on MWCNT synthesis by gradually increasing the amount of $\mathrm{H}_{2} \mathrm{SO}_{4}$ in solution with $\mathrm{H}_{3} \mathrm{PO}_{4}$ used for the

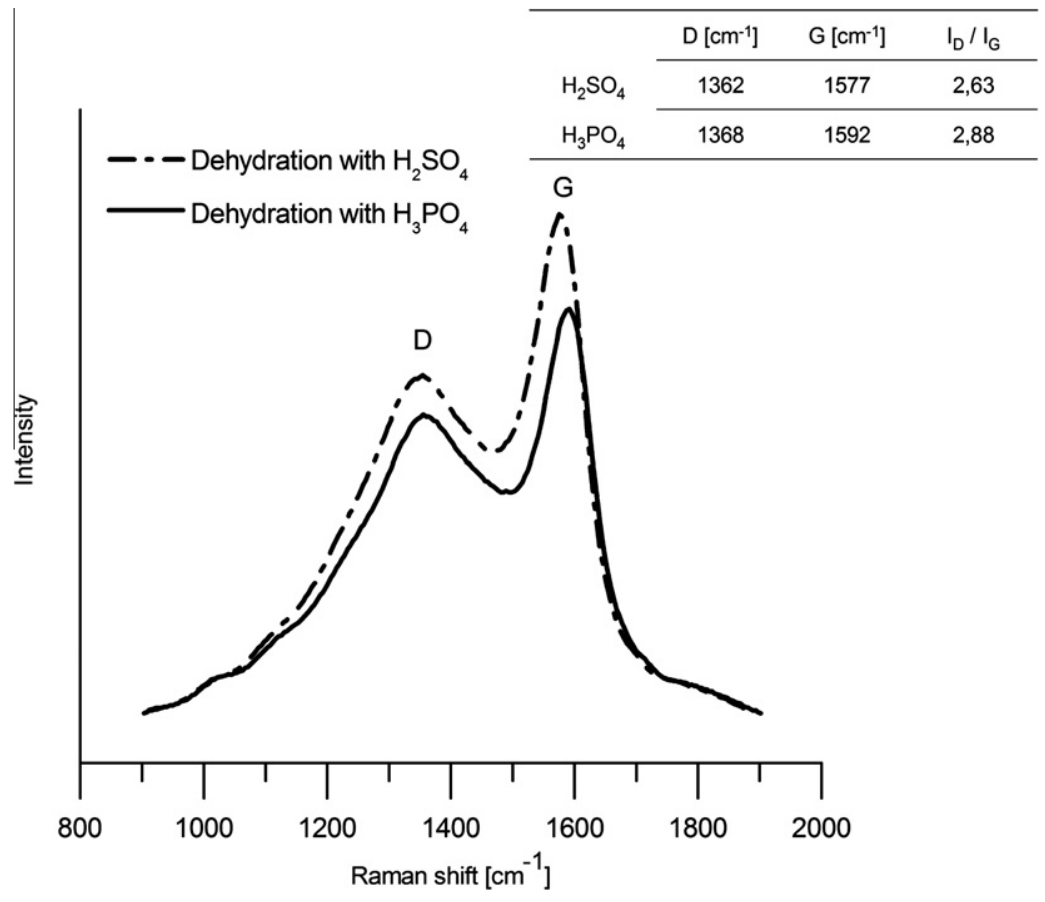

Fig. 4 - Comparison of the Raman spectra from carbonized matter dehydrated with $\mathrm{H}_{2} \mathrm{SO}_{4}$ and $\mathrm{H}_{3} \mathrm{PO}_{4}(1: 1 \mathrm{Fe}$ and $\mathrm{Ni}$ catalyst mixture). The inset shows tabulated data derived from the spectra and the intensity ratios are calculated using integrated intensities for $D$ and $G$ peaks. 

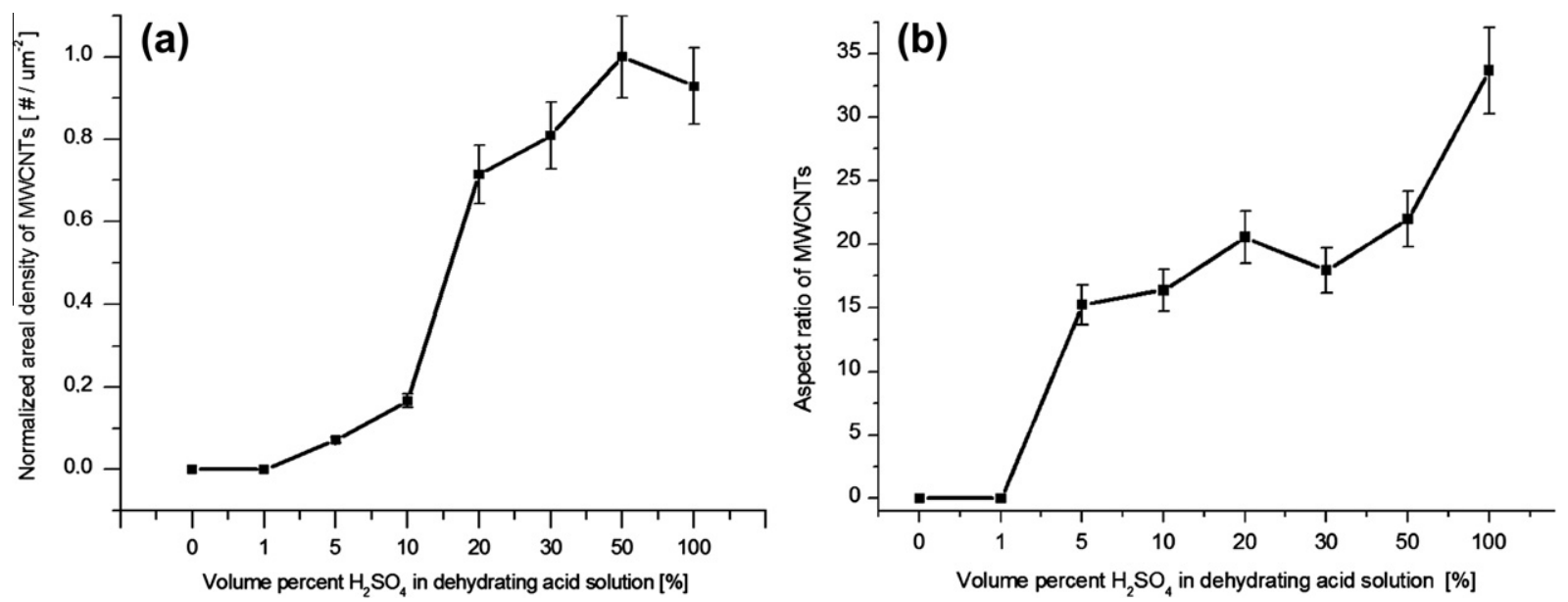

Fig. 5 - (a) A plot showing the normalized areal density of MWCNTs observed versus the volume percentage $\mathrm{H}_{2} \mathrm{SO}_{4}$ used for carbonization. (b) A plot for the aspect ratio of MWCNTs against volume percentage $\mathrm{H}_{2} \mathrm{SO}_{4}$ used for carbonization.

carbonization of the sucrose-catalyst mixture; $50 \%, 30 \%, 20 \%$, $10 \%, 5 \%$ and $1 \%$ by volume solutions of $\mathrm{H}_{2} \mathrm{SO}_{4}$ were used. In an attempt to quantify the sulfur effect, we have carefully counted the number of MWCNTs from randomly captured SEM images for each run and calculated an areal density of MWCNTs for each corresponding experiment. Fig. 5a shows a plot for the normalized areal density of MWCNTs observed versus the volume percentage $\mathrm{H}_{2} \mathrm{SO}_{4}$ used for carbonization. At around $30 \mathrm{vol} . \% \mathrm{H}_{2} \mathrm{SO}_{4}$, a sharp increase in the areal density was observed. While, both Kim et al. [31], and Tibbetts et al. [32] reported the eventual termination of carbon fiber growth beyond a certain level of sulfur, we did not observe a drop in MWCNT areal density for the run with pure $\mathrm{H}_{2} \mathrm{SO}_{4}$ case. Also, we have investigated the effect of $\mathrm{H}_{2} \mathrm{SO}_{4}$ on the size of the MWCNTs by the plotting the aspect ratio of MWCNTs against volume percentage $\mathrm{H}_{2} \mathrm{SO}_{4}$ used for carbonization, shown in Fig. 5b. The results indicated although the amount of sulfur had a strong effect on the synthesis of MWCNTs and their population density, it did not seem to alter the size and shape of MWCNTs notably, unless pure $\mathrm{H}_{2} \mathrm{SO}_{4}$ was used.

Investigation of the local chemistry in and around the MWCNTs formed with pure $\mathrm{H}_{2} \mathrm{SO}_{4}$ employing EDS during TEM investigations provided valuable clues regarding the details of how sulfur affects CNT growth. As mentioned earlier, a number of studies link the observation of Y-junctions in CNTs to the presence of sulfur in the side-wall chemistry $[7,12,33]$. We analyzed the chemistry of the CNT side-walls and the catalyst particles using EDS technique during TEM analysis. We did not observe presence of any sulfur in the CNT side walls, as shown in Fig. 6a. There was also no indication of a change in the CNT morphology in response to varying the sulfur amount. Although, we were not able to detect presence of sulfur in the side-walls of CNTs, EDS data indicated significant amount of sulfur incorporated inside the metal catalyst particles, as shown in Fig. 6b. The atomic ratio of sulfur incorporated with Fe was found to be nearly 1:1. Similar observations indicating large amounts of sulfur in the catalyst particles has been reported by Demoncy et al. [34]. Furthermore, in a recent study researchers showed rapid growth of CNTs on a molten Fe and sulfur containing catalyst particle. In addition, it was argued that the molten particle was actually a phase mixture of two immiscible liquids and a solid phase; $\mathrm{L}_{1}$, sulfur-rich liquid phase, $\mathrm{L}_{2}$, carbon rich liquid phase and carbon as the solid phase in the Fe-S-C ternary phase diagram [35]. In this proposed growth model, the sulfur-rich molten layer was thought to be facilitating the CNT growth. While this proposed view is inline with our observations regarding catalyst particles with very high sulfur content facilitating CNT growth and may be extrapolated to explain the effect of sulfur on the population density and aspect ratio of MWCNTs, it still does not provide any lead for the explanation of why some researchers observed sulfur in the vicinity of Yjunctions at the CNT side-walls, or how sulfur may induce Y-junction formation.

To provide detailed understanding of our experimental observations at an atomistic level, we have performed DFT calculations exploring the interaction of sulfur and hexagonal carbon network mimicking the atomic structure of CNT sidewall. In the first part of these studies, we have introduced a single sulfur atom on a non-defective graphene-based sheet. Upon relaxation of the geometry with regards to total energy, we observed that the sulfur adatom situated itself at the valley between two adjacent carbon atoms, effectively chemically bonding to both carbon atoms as shown in Fig. 7a, with a sulfur-carbon bond length of $1.85 \AA$. When a second sulfur atom was introduced to the system, energetically most favorable condition formed by the dimerization of the two sulfur atoms, see Fig. 7b. An unexpected outcome of this was the fact that the distance between the sulfur dimer and the underlying carbon network significantly increased with respect to single sulfur adatom case, pointing a weaker interaction between the sulfur and carbon atoms: sulfur-carbon bond length increased to $3.57 \AA$, while the sulfur-sulfur interaction got stronger. In both Fig. $7 \mathrm{a}$ and $\mathrm{b}$, it can be seen that presence of the sulfur adatoms on the graphene layer did not induce a significant distortion; hence sulfur adsorption by itself cannot cause large scale structural deformations in the CNT side-wall network. 

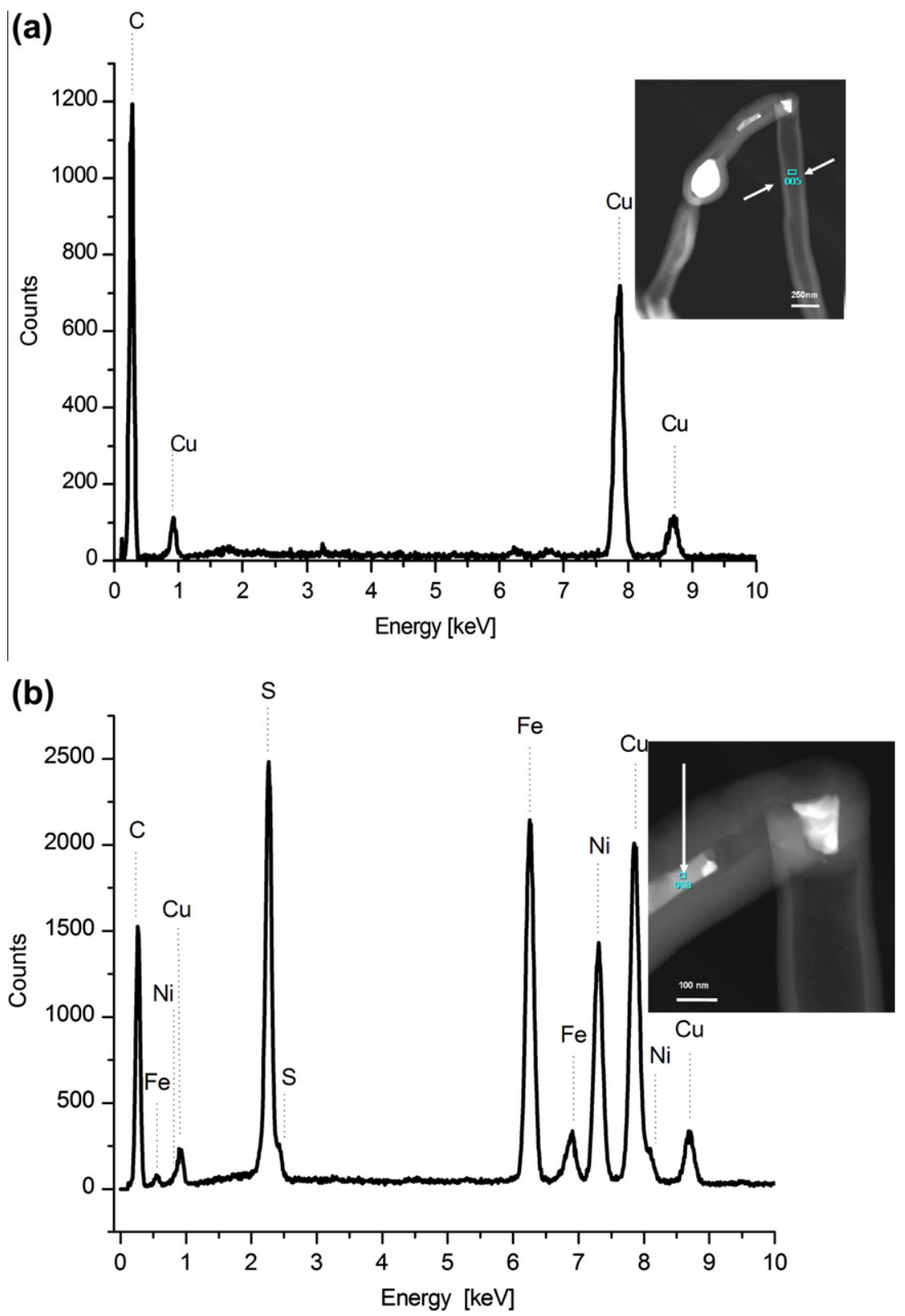

Fig. 6 - (a) EDS analysis on the side walls of MWCNT showing no signal for sulfur. (b) EDS analysis on the metal catalyst of Fe and $\mathrm{Ni}$ mix showing the presence of sulfur with Fe in atomic ratio 1:1. Insets indicate locations for EDS data collection. (Cu is signal is due to the $\mathrm{Cu}$ based TEM grid used.)

We examined the case of saturation of a vacancy in the hexagonal carbon network with a single sulfur atom, as well. Presence of a sulfur atom as a substitutional in the carbon hexagonal ring network induced some strain locally as shown in Fig. 7c, yet this was far from providing a lead toward explaining the evolution of junction geometry due to sulfur presence in the CNT sidewalls, such as one shown in the "opening branch" locations by Romo-Herrera et al. [12].

It is well known that capping of CNTs require the presence of non-hexagonal carbon rings following Euler's Law, especially pentagonal ones. In our earlier study dealing with the nano-arch geometry in BN structures, we found that nucle- ation of non-hexagonal rings lead to the formation of highly strained non-planar geometries [36]. Motivated with this, we have investigated several non-hexagonal defect structures and their interaction with sulfur atoms in a planar graphene layer. However, in this paper we limit our discussion to the most energetic cases for the non-hexagonal defects; a single pentagonal defect and conjoined double pentagonal defects as shown in Fig. 8a and b, respectively. As shown in these figures, the curvature induced to the graphene sheet increases with the number of pentagonal defects forced into the graphene structure. In this respect, reconsidering the emerging branch geometry observed in Fig. 2a of [12] and arguments therein regarding atomic positions sulfur may assume in the 


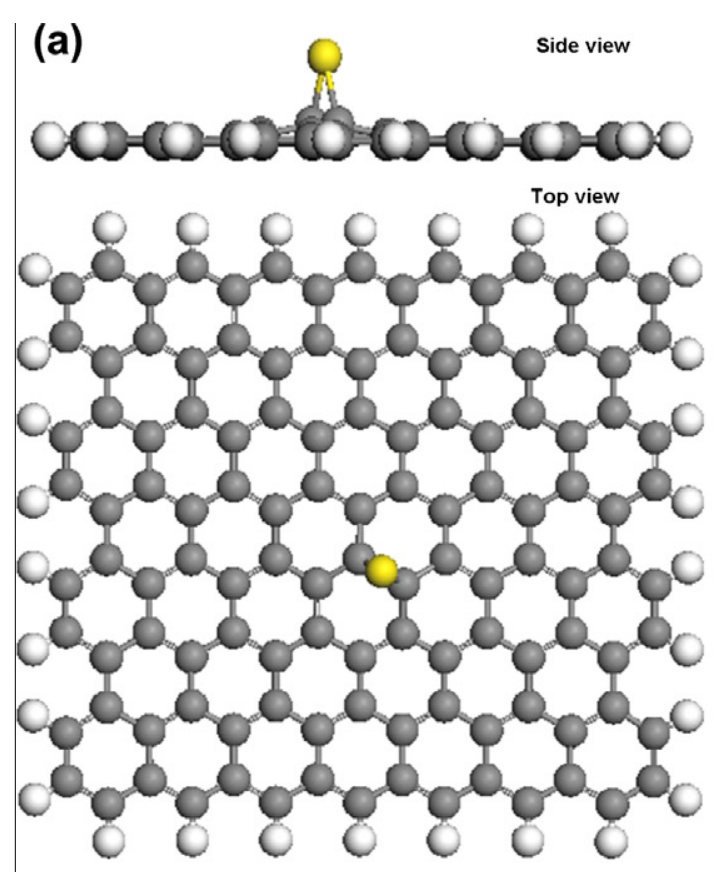

(c)
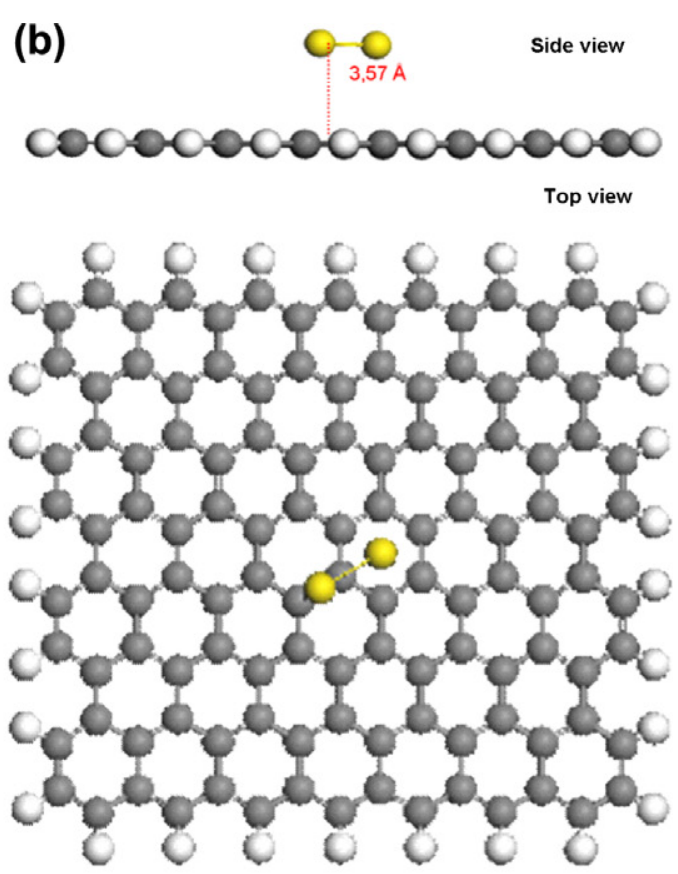

Side view
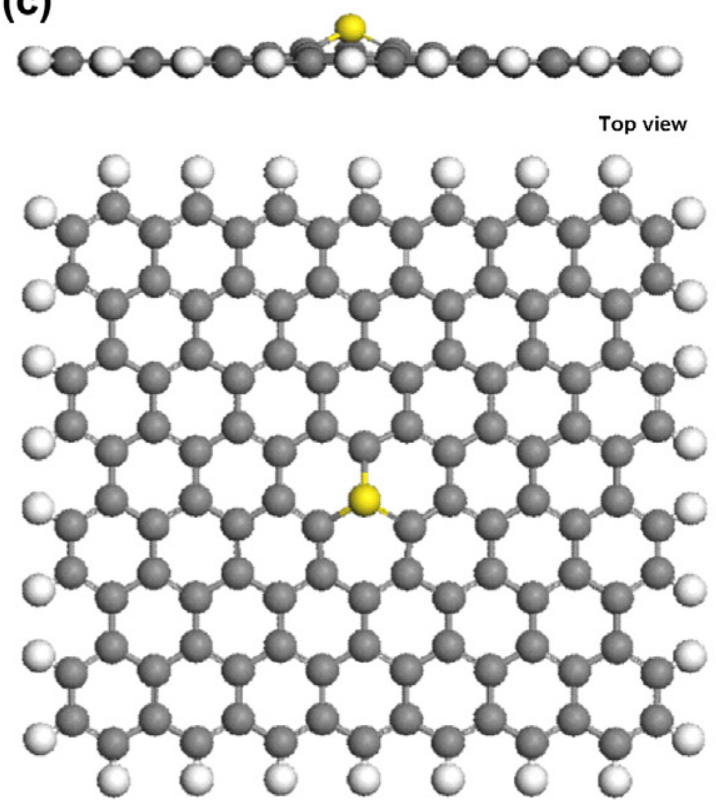

Fig. 7 - DFT calculations showing the relaxed forms of structures representing possible position of sulfur absorbing on several different structures (a) single sulfur atom on graphene sheet, (b) two sulfur atoms on graphene sheet, (c) single sulfur as a substitutional defect in hexagonal carbon network.

CNT geometry, we decided to investigate the interaction between a sulfur adatom and carbon atoms in a non-hexagonal ring. We propose that regions in the CNT side-walls with one or more non-hexagonal rings can provide the curvature needed for forming Y-junctions. Therefore, we believe that such defects act as nucleation sites for such structures.

The relaxed defective structures used to study the energetics of sulfur-carbon interaction for a single pentagonal defect and conjoined double pentagonal defects are displayed in Fig. $8 c$ and d, respectively. We calculated the binding energy per sulfur adatom for these structures and these are listed in Table 2. The outcome of our calculations on these structures suggested several important points;

(1) Sulfur preferred defective regions as binding sites where carbon atoms form non-hexagonal rings.

(2) The interaction between the sulfur and carbon atoms remained strong for defective structures, even when sulfur dimerized as shown in Fig. $8 \mathrm{c}$ and d. In the case of sulfur binding to a carbon atom in a hexagonal ring, the 

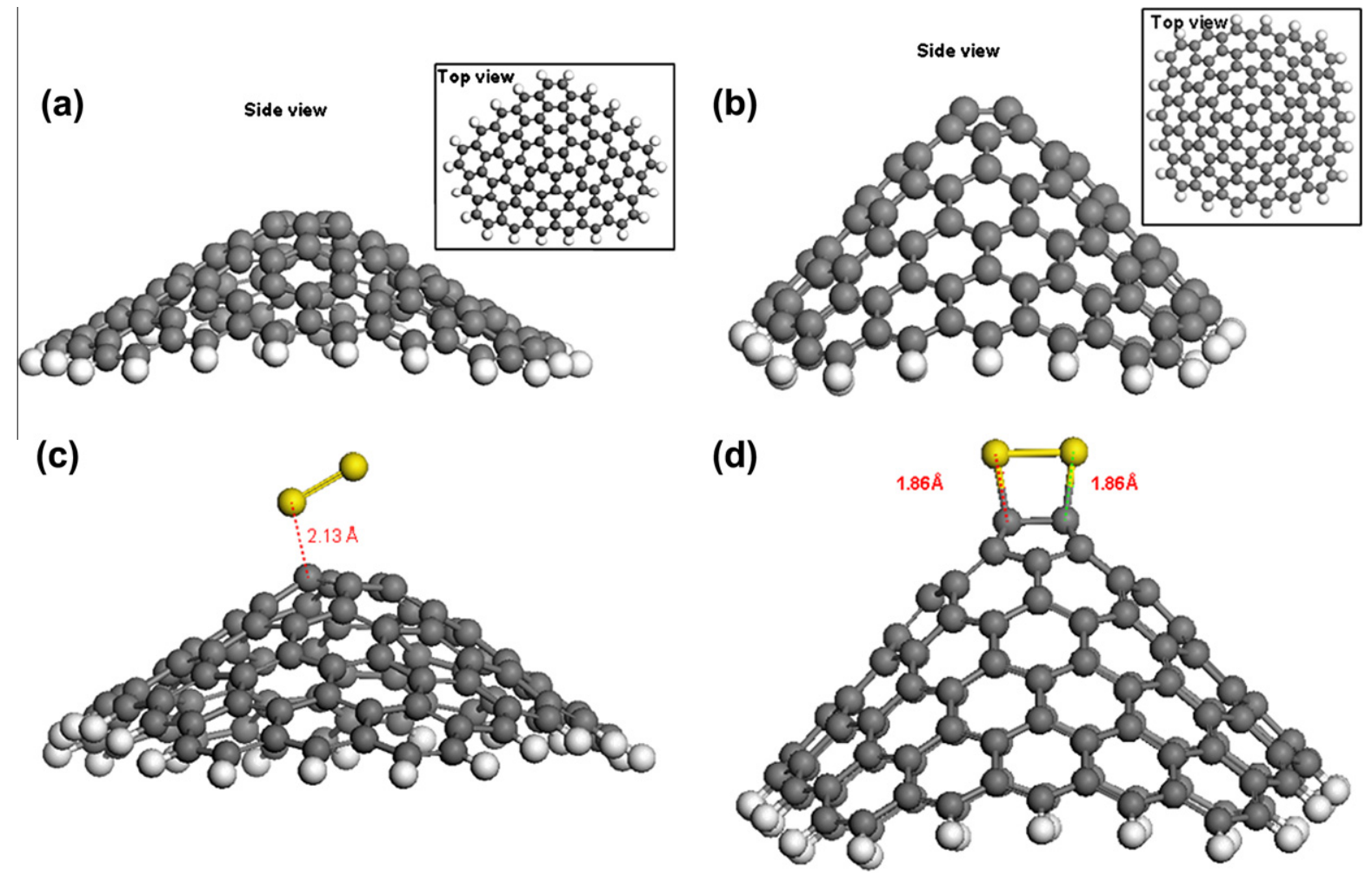

Fig. 8 - Results from DFT calculations showing the relaxed defective structures (top and side views); (a) a single pentagonal defect, (b) conjoined double pentagonal defect, (c) after binding of a sulfur dimer to a single pentagonal defect and (d) to a conjoined double pentagonal defect.

\section{Table 2 - Tabulated values of binding energy per atom are shown for different atomic sulfur containing structures.}

\begin{tabular}{lc}
\hline Defect type & $\begin{array}{c}\text { Binding energy/ } \\
\text { atom }^{\mathrm{a}}(\mathrm{eV})\end{array}$ \\
\hline $\begin{array}{l}\text { A single S adatom on non-defective } \\
\text { hexagonal carbon network }\end{array}$ & -1.69 \\
$\begin{array}{l}\text { Two individually bonded S } \\
\text { adatoms on non-defective } \\
\text { hexagonal carbon network }\end{array}$ & -1.79 \\
S dimer on non-defective \\
hexagonal carbon network \\
$\begin{array}{l}\text { A single S adatom on a } \\
\text { pentagonal defect }\end{array}$ \\
$\begin{array}{l}\text { A single S adatom on aconjoined } \\
\text { pentagonal defect }\end{array}$ \\
$\begin{array}{l}\text { S dimer on a pentagonal defect } \\
\text { S dimer on a conjoined } \\
\text { pentagonal defect }\end{array}$ \\
$\begin{array}{l}\text { a Binding energy is calculated as follows: } E(\text { Binding })=E(C+H+S)- \\
E(C+H)-E(S) .\end{array}$ \\
\hline
\end{tabular}

interaction between sulfur and carbon gets significantly weaker, if sulfur forms a dimer with another sulfur atom in the vicinity.

(3) None of the atomic models examined in this study indicated a significant change in the pre-existing curvature of the hexagonal carbon network due to binding of sulfur. Thus, sulfur is not likely to induce a pentagonal defect in the hexagonal carbon network.
Conceivably, both the experimental data and results of the theoretical studies presented here pose a contradictory statement with regards to the studies noting a relationship between sulfur and Y-junction presence in CNTs. While, we have not observed Y-junction geometries in our experiments that were run under sulfur rich conditions, the results of our calculations also suggested sulfur cannot be the cause for the branching mechanism. However, one plausible explanation for the observation of sulfur at an emerging branch by Romo-Herrera et al. [12] could be that sulfur prefers binding with carbon atoms at defective regions as our DFT calculations suggested.

Moreover, there are reports in the literature regarding branching of CNTs, where there were no apparent involvement of sulfur in the reaction environment $[37,38]$. Deepak et al. [8] offered the combined effect of several process parameters for the explanation of the Y-junction morphology even with presence of sulfur in the reactant mixture. These reports support our explanation of the lack of Y-junctions and non-existence of sulfur in the CNT side-walls for our study. However, there are other factors that need to be taken into account. First of all, the synthesis temperature we have used can be considered as unusually high for CNT formation. It is likely that at these temperatures, some of the non-hexagonal rings and other defects in the side-wall structures could be annealed decreasing the possibility for the nucleation of Yjunctions. Hence, if sulfur atoms are unable to find suitable defect sites for binding, they will tend to form dimers or even larger clusters further weakening their interaction with the 
underlying carbon network. Eventually, sulfur dimers or clusters can easily break free and leave the carbon network in gaseous form. This can explain the lack of sulfur we have observed in the side-wall chemistry of MWCNTs we have analyzed.

\section{Conclusion}

We investigated effect of sulfur on CNT growth and morphology, especially Y-branching mechanism, through a new technique where MWCNTs were synthesized by the pyrolysis of $\mathrm{H}_{2} \mathrm{SO}_{4}$ carbonized byproduct of sucrose. We have demonstrated that sulfur presence was crucial for the CNT formation and for increasing the population density in the post-pyrolysis residue, but we did not observe any Y-junctions. Also, we did not encounter any sulfur in the side-walls of the CNTs formed, while a significant amount of sulfur was found in the catalyst particles. The results of our DFT calculations showed that sulfur is not by itself capable of generating defects or inducing curvatures required for the nucleation of a Y-branch, and thus cannot be the initiator for a Y-junction. On the other hand, our data indicated that sulfur prefers to bind to readily existing non-hexagonal defects, and we suggest that this is the underlying factor for some studies reporting presence of sulfur at the emerging branch sites. Finally, we believe that non-hexagonal defects are the key for the formation of Y-junctions as they act as nucleation sites regardless of sulfur binding to them. Therefore, control and understanding the formation of non-hexagonal defects and their role in Y-junction formation during synthesis of CNTs is a key step in the fabrication of robust 3D networks of CNTs.

\section{Acknowledgements}

The authors would like to acknowledge financial support for the work by TUBITAK (Grant Nos.: 109 T026 and 107T720) and European Union 7. Framework project Unam-Regpot (Grant No.: 203953). The Center for Electron Microscopy and Microanalysis at the Metallurgical and Materials Engineering Department of METU is also acknowledged for assistance with TEM imaging.

\section{R E F E R E N C E S}

[1] Romo-Herrera JM, Terrones M, Terrones H, Dag S, Meunier V. Covalent 2D and 3D networks from 1D nanostructures: designing new materials. Nano Lett 2007;7(3):570-6.

[2] Scuseria GE. Negative curvature and hyperfullerenes. Chem Phys Lett 1992;195(5-6):534-6.

[3] Chernozatonskii L. Carbon nanotube connectors and planar jungle gyms. Phys Lett A 1992;172(3):173-6.

[4] Andriotis AN, Menon M, Strivastava D, Chernozatonskii L. Rectification properties of carbon nanotube "Y-junctions". Phys Rev Lett 2001;87(6):066802.

[5] Meunier V, Nardelli MB, Bernholc J, Zacharia T. Intrinsic electron transport properties of carbon nanotube Yjunctions. Appl Phys Lett 2002;81(27):5234-6.

[6] Bengu E, Marks LD. Single-walled BN nanostructures. Phys Rev Lett 2001;86(11):2385-7.
[7] Satishkumar BC, Thomas PJ, Govindaraj A, Rao CNR. Yjunction carbon nanotubes. Appl Phys Lett 2000;77(16):2530-2.

[8] Deepak FL, Govindaraj A, Rao CNR. Synthetic strategies for Yjunction carbon nanotubes. Chem Phys Lett 2001;345(12):5-10.

[9] Zhu H, Ci L, Xu C, Liang J, Wu D. Growth mechanism of Yjunction carbon nanotubes. Diamond Relat Mater 2002;11(7):1349-52.

[10] Wang Z, Zhao Z, Qiu J. Synthesis of branched carbon nanotubes from coal. Carbon 2006;44(7):1321-4.

[11] Ma H, Wang Y, Jin G, Guo X. Structural changes in carbon produced by a sulfur-aided catalytic chemical vapor deposition. New Carbon Mater 2009;24(1):13-7.

[12] Romo-Herrera JM, Sumpter BG, Cullen DA, Terrones H, CruzSilva E, Smith DJ, et al. An atomistic branching mechanism for carbon nanotubes: sulfur as the triggering agent. Angew Chem Int Ed Engl 2008;47(16):2948-53.

[13] Yu JS, Yoon SB, Chai GS. Ordered uniform porous carbon by carbonization of sugars. Carbon 2001;39:1442-6.

[14] Franklin RE. Crystallite growth in graphitizing and nongraphitizing carbons. Proc Roy Soc A 1951;209:196-218.

[15] Harris PJF, Tsang SC. High-resolution electron microscopy studies of non-graphitizing carbons. Philos Mag A 1997;76(3):667-77.

[16] Setlur AA, Doherty SP, Dai JY, Chang RPH. A promising pathway to make multiwalled carbon nanotubes. Appl Phys Lett 2000;76(21):3008-10.

[17] Kumar M, Ando Y. Chemical vapor deposition of carbon nanotubes: a review on growth mechanism and mass production. J Nanosci Nanotechnol 2010;10(6):3739-58.

[18] Abdel-Fattah T, Siochi EJ, Crooks RE. Pyrolytic synthesis of carbon nanotubes from sucrose on a mesoporous silicate. Fullerenes, Nanotubes, Carbon Nanostruct 2006;14(4):585-94.

[19] Payne MC, Teter MP, Allen DC, Arias TA, Joannopoulos JD. Iterative minimization techniques for ab-initio total-energy calculations: molecular dynamics and conjugate gradients. Rev Mod Phys 1992;64(4):1045-97.

[20] Kohn W, Sham LJ. Self-consistent equations including exchange and correlation effects. Phys Rev 1965;140(4A):A1133-8.

[21] Hohenberg P, Kohn W. Inhomogeneous electron gas. Phys Rev 1964;136(3B):B864-71.

[22] Blöchl PE. Projector augmented-wave method. Phys Rev B 1994;50(24):17953-79.

[23] Kresse G, Joubert D. From ultrasoft pseudopotentials to the projector augmented-wave method. Phys Rev B 1999;59(3):1758-75.

[24] Kresse G, Hafner J. Efficiency of ab-initio total energy calculations for metals and semiconductors using a planewave basis set. Phys Rev B 1993;48(17):13115-8.

[25] Kresse G, Furthmüller J. Efficiency of ab-initio total energy calculations for metals and semiconductors using a planewave basis set. Comput Mater Sci 1996;6(1):15-50.

[26] Kresse G, Furthmüller J. Efficient iterative schemes for abinitio total-energy calculations using a plane-wave basis set. Phys Rev B 1996;54(16):11169-86.

[27] Perdew JP, Chevary JA, Vosko SH, Jackson KA, Pederson MR, Singh DJ, et al. Atoms, molecules, solids, and surfaces: applications of the generalized gradient approximation for exchange and correlation. Phys Rev B Condens Matter 1992;46(11):6671-87.

[28] Monkhorst HJ, Pack JD. Special points for Brillouin-zone integrations. Phys Rev B 1976;13(12):5188-92.

[29] Robertson J. Diamond-like amorphous carbon. Mater Sci Eng R 2002;37:129-281.

[30] Tibbetts G. Why are carbon filaments tubular. J Cryst Growth 1984;66(3):632-8. 
[31] Kim MS, Rodriguez NM, Baker RTK. The interplay between sulfur adsorption and carbon deposition on cobalt catalysts. J Catal 1993;143(2):449-63.

[32] Tibbetts GG, Bernardo CA, Gorkiewicz DW, Alig RL. Role of sulfur in the production of carbon fibers in the vapor phase. Carbon 1994;32(4):569-76.

[33] Valles C, Perez-Mendoza M, Castell P, Martinez MT, Maser WK, Benito AM. Towards helical and Y-shaped carbon nanotubes: the role of sulphur in CVD processes. Nanotechnology 2006;17(17):4292-9.

[34] Demoncy N, Stephan O, Bran N, Colliex C, Loiseau A, Pascard H. Sulfur: the key for filling carbon nanotubes with metals. Synth Met 1999;103(1-3):2380-3.
[35] Motta MS, Moisala A, Kinloch IA, Windle AH. The role of sulphur in the synthesis of carbon nanotubes by chemical vapour deposition at high temperatures. J Nanosci Nanotechnol 2008;8(5):2442-9.

[36] Bengu E, Marks LD, Ovali RV, Gulseren O. Analysis of defects on BN nano-structures using high-resolution electron microscopy and density functional calculations. Ultramicroscopy 2008;108(11):1484-9.

[37] Li WZ, Wen JG, Ren ZF. Straight carbon nanotube Y-junctions. Appl Phys Lett 2001;79(12):1879-81.

[38] Ding DY, Wang JN, Yu F, Su LF. Influence of pyrolysis temperature on the growth of Y-junction carbon nanotubes. Appl Phys A 2005;81(4):805-8. 